\title{
Metabolomics in the natural products field - a gateway to novel antibiotics
}

\section{Changsheng $\mathrm{Wu}^{1,2}$, Hye Kyong Kim', Gilles P. van Wezel ${ }^{2}$, Young Hae Choi ${ }^{1, *}$}

'Natural Products Laboratory, Institute of Biology, Leiden University, Sylviusweg 72, 2333 BE Leiden, The Netherlands

${ }^{2}$ Molecular Biotechnology, Institute of Biology, Leiden University, Sylviusweg 72, 2333 BE Leiden, The Netherlands

\begin{abstract}
Metabolomics is a high throughput analytical technique used to globally measure low molecular weight metabolites, allowing simultaneous metabolic comparison of different biological samples and thus highlighting differentially produced compounds as potential biomarkers. Although microbes are renowned as prolific sources of antibiotics, the traditional approach for new anti-infectives discovery is time-consuming and labor-intensive. In this review, the use of NMR- or MS-based metabolomics is proposed as an efficient approach to find antimicrobials in microbial single- or co-cultures.
\end{abstract}

\section{Introduction}

The number of multi-drug resistant bacteria is rising alarmingly and the treatment of infections caused by these microbes is extremely challenging [1]. Actinomycetes and filamentous fungi are the major source of the natural antibiotics. The traditional methods used for drug discovery is time- and labor-intensive and the rediscovery rates became increasingly demotivating [2,3]. Yet, genome sequencing revealed many previously unsuspected biosynthetic clusters for natural products in long-studied model strains [4-6]. A major challenge lies in finding the appropriate chemical triggers or ecological cues to elicit the production of cryptic antibiotics $[7,8]$.

Metabolomics is a high throughput analytical technique used to globally measure low molecular weight metabolites in

*Corresponding author: Y.H. Choi (y.choi@chem.leidenuniv.nl)

\section{Section editor:}

Pascal de Tullio - University of Liège, Liège, Belgium.

biological samples. Combination of chemical profiles built by LC-HRMS or NMR with multivariate data analysis (MDA) allows scientists to compare and detect differential metabolites in active/inactive biological samples, narrowing the search to potential biomarkers and avoiding chemical redundancy in an early stage. In this paper, we have reviewed examples of the use of metabolomics as an effective tool to increase the efficiency of antibiotic discovery from microbial sources.

\section{Metabolomics-guided discovery of new antibiotics}

Metabolomics methodology, either NMR- or MS-based, is an ideal tool for the chemical screening and subsequent detailed comparison of the secondary metabolomes of a set of bacterial fermentations, rapidly revealing the differences/biomarkers among experimental groups. The next question is how to handle the compounds responsible for the separation in the score plot of supervised and/or unsupervised MDA. Because NMR and MS techniques can give structural information of compounds in an early stage, there are three possibilities for the identification of valuable biomarkers. Firstly, it is possible to compare the structural information provided by NMR $\left({ }^{1} \mathrm{H}\right.$ NMR chemical shifts) and/or high-resolution mass spectrometry (molecular formula and isotopic pattern) with literature and/or databases. If known structures, discriminators can be identified without the need for time-consuming isolation process and thus metabolomics serves as an approach for 
dereplication; alternatively, new compounds may possess known structural core but unknown functional groups 'known unknowns'. In this case, their structural characterization can be done within the context of the crude biological matrix with the aid of various $2 \mathrm{D}$ NMR techniques as reviewed in $[9,10]$. Besides this, the fragmentation patterns observed in the MS/MS spectra in conjunction with molecular networking evaluation can enable the identification of previously unknown close relatives within the same chemical families through spectral correlations $[11,12]$. Finally, if the compounds have unprecedented nuclei, chromatographic isolation is inevitable for full de novo NMR structural characterization, but at the same time are the most promising in terms of truly new antimicrobials discovery. For this step, the discriminating signals generated in the comparative analysis (MDA) could be used as probes to track the target of interest ('star antibiotic') throughout the chromatographic isolation, which is referred to as NMR- $[13,14]$ and/or MS-guided $[15,16]$ separation method. This can prevent losing track of the target that is unfortunately quite common in conventional TLCguided isolation. Furthermore, some degree of structure identification, albeit incomplete, can be achieved beforehand with the 2D NMR measurements because they allow a partial prediction of the novel compounds. This can also provide information on chemical characteristics and help optimize isolation procedure, which makes senses for minimizing the loss of compounds (e.g. ruling out the use of bare silica gel for polyphenolic polyketides due to their practically irreversible adsorption as in the case of clostrubin [17]). Taken together, the strategy of metabolomics-guided discovery of new antibiotics is illustrated in Scheme 1.

The use of metabolomics as a methodology for de-replication of known microbial secondary metabolites and prioritization of promising candidates is exemplified in the following cases. The LC-MS metabolome profiling of nine Myxococcus species allowed the rapid identification of known myxobacterial metabolites and the subsequent compoundbased principal component analysis (PCA) was successfully highlighted the putative novel compound determined by its exact mass and isotopic pattern [18]. Tim Bugni and colleagues [19] used UHPLC-HRMS-based metabolomics to dereplicate known structures and predict novel compounds in microbial crude extract. They then applied MS-guided separation approach to target the molecules highlighted by metabolomics analysis, and successfully discovered a series of antibiotics with novel skeletons, such as microtermolides [20], bottromycin D [21] and more recently forazoline A [22]. Forazoline A (Fig. 1) is a potent polyketide with a highly unusual skeleton, exhibiting in vivo antifungal efficacy against Candida albicans (MIC $16 \mu \mathrm{g} / \mathrm{mL}$ ) - comparable to that of amphotericin B in a mouse model of C. albicans and no obvious cytotoxicity [22]. Furthermore, they utilized a LCMS-SPE-NMR hyphenation system that integrates MS-guided separation and de novo NMR structure elucidation [23] to facilitate rapid structure elucidation of rare phenyl-acetyldesferrioxamines indicated by metabolomics analysis [19]. In case of lowly abundant compounds, targeted LC-MS microisolation strategy reported by Jean-Luc Wolfender and colleagues [24,25] for the purification of novel metabolites is necessary for full structure determination by means of microflow NMR analysis that just requires micrograms of compounds $[26,27]$.

\section{Metabolomics applied to microbial cocultivation}

Conventionally, scientists isolate pure strains of microbes collected from various biological samples, such as soil, marine sediments, symbionts of plants or sponges. Fermentations are then scaled up to accumulate sufficient material for the next-stage systematical or bioassay-guided isolation. However, the isolation of novel antibiotics from microbes by means of this well-established routine is difficult. One of the reasons is many putative gene clusters remain silent under standard laboratory growth conditions when microbes are cultivated singly. Thus, it might be fruitful to consider not only single microorganism but more complex biological systems consisting of different organisms. This novel approach in which microbes are grown together (co-culture or confrontation experiments) has received increasing interest for its potential to produce new leads, and to decipher specific biosynthetic pathways mainly related to defense [28]. The focus should be set on antagonistic interactions among the different microbes (fungi and/or bacteria) coexisting in the same defined environment, because they use chemical warfare consisting generally of small organic compounds for defense. In some cases, clear long-distance growth inhibition can be observed during pairwise co-culture on solid media. This phenomena is also expected to be linked to the production of antimicrobial compounds as a defense mechanism by one of the two co-inhabiting microorganisms $[25,29]$. Coculturing the fungal endophyte Fusarium tricinctum with the bacterium Bacillus subtilis 168 trpC2 on solid rice medium resulted in up to 78 times the production of enniatins A1 and B1 [30]. Microbial cocultivation is also effective for the induction of new molecules with antimicrobial effect, as verified by the discovery of the new antibiotics pestalone [31], emericellamides A and B [32], O-methylmellein [33]. It is quite probably that one microbe produce molecules that could be precursors for biotransformation into other final products by its partner, which can increase the chemodiversity of induced metabolites and thus the chance of finding novel antibiotic [34].

Considering the great potential of cocultivation as a fertile source for new antibiotics, it is necessary to develop effective methods for their detection and identification of induced metabolites. Metabolomics is particularly appropriate for this purpose as indicated in [29]. Untargeted generic 


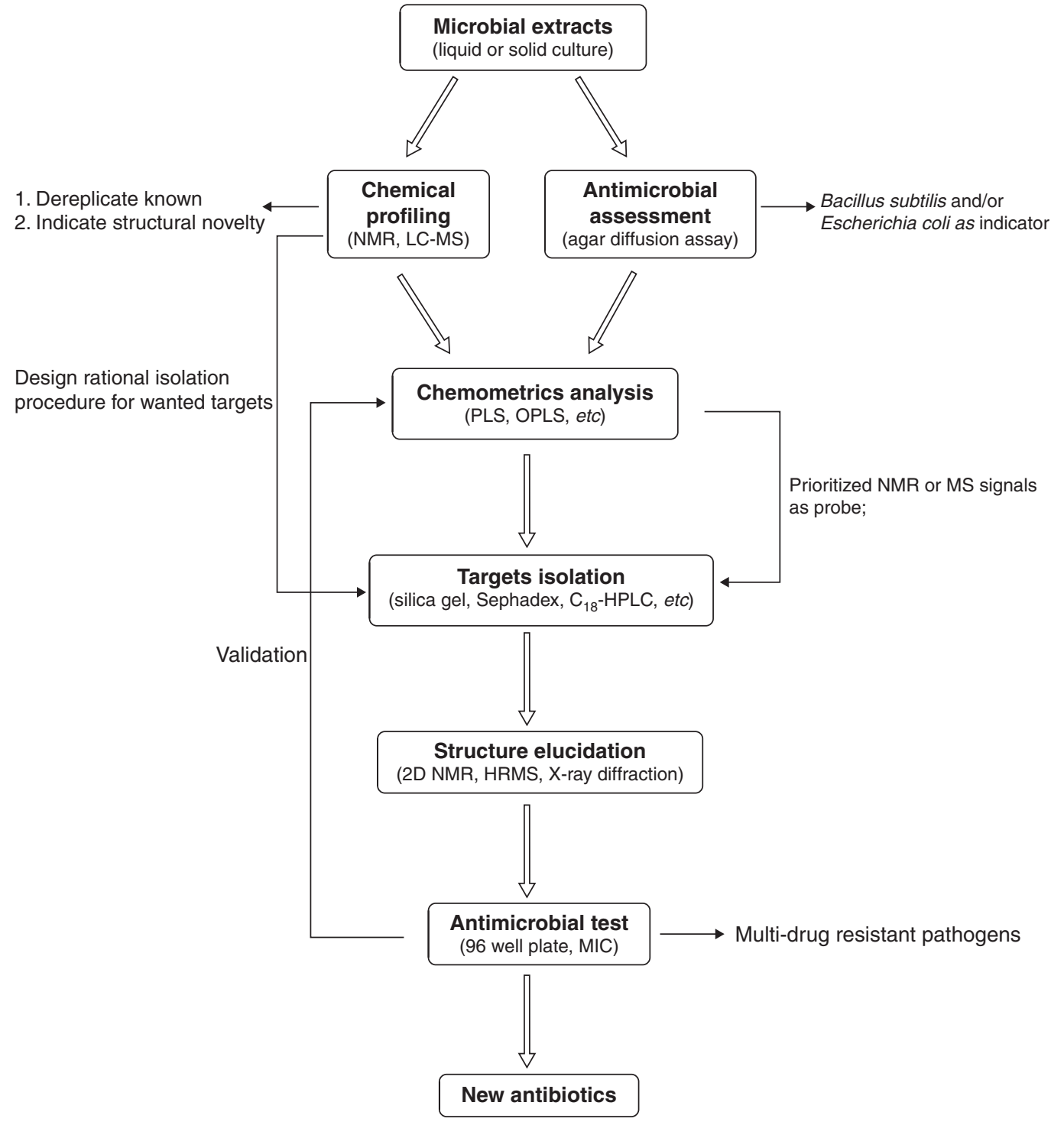

Drug Discovery Today: Technologies

Scheme I. Metabolomics-guided discovery of new antibiotics.<smiles>COC(=O)C[C@H](NC(=O)[C@H](CC(=O)C(N=C1NCC(=O)N2CC[C@H](C)[C@H]2C(=O)N[C@@H](C)C(=O)N[C@@H]1C(C)(C)C)C(C)(C)C)[C@@H](C)c1ccccc1)c1nccs1</smiles>

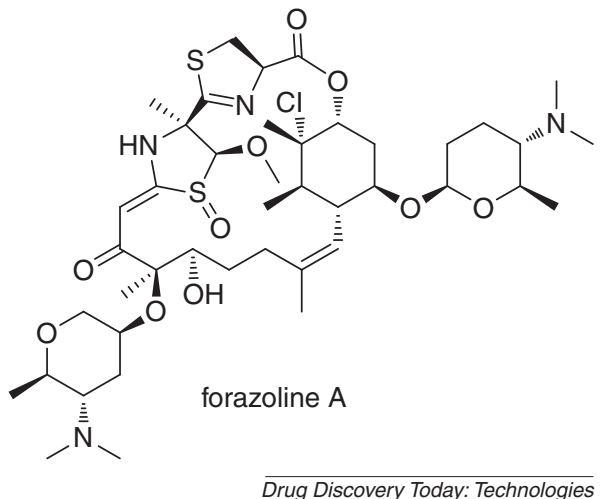

Figure I. Structurally novel antibiotics discovered through the framework of metabolomics methodology. 
fingerprinting using ${ }^{1} \mathrm{H}$ NMR, but more by hyphenated MS methods (considered by some to be more powerful because of their sensitivity) is aimed to capture metabolites comprehensively. The comparison of the metabolome of the coculture with that of the individual microorganisms can highlight the induced metabolites present in coculture but absent in monocultures, because these de novo-produced molecules have high chances of being new compounds (or even new skeletons) with antimicrobial activity. However, it is important to bear in mind that the conventional chemometric methods, such as PCA and (O)PLS-DA, are not absolutely ideal for coculture metabolomics studies, because they might not effectively filter the up-regulated metabolites in monocultures and thus not always highlight the cocultureinduced metabolites specifically. A novel data mining approach referred to as projected orthogonalized chemical encounter monitoring (POChEMon) was recently developed and has proved to be more appropriate for this purpose [28].

The detection of antibiotics using the microbial cocultivation strategy may be further revolutionized by the application of advanced analytical technologies introduced into metabolic profiling, one of which is imaging mass spectrometry (IMS) [35-37]. This technique can provide distinct chemical/ structural data on the spatial distributions of metabolites directly on the agar Petri dishes avoiding complex sample pre-treatment [38-41]. For this, the antagonistic interaction between tested microorganisms is favored. After the antagonistic phenotypes are observed, the inhibition regions are subjected to IMS screening to visualize the metabolites. The microbial metabolites with a spatial distribution that is superimposed with the zone of inhibition are considered to be potential anti-infective candidates. The exact mass and isotope pattern provided by high resolution instruments such as MALDI-TOF-IMS, in combination with MS/MS network analysis can help estimate the structural novelty of the induced metabolites $[11,42]$. This technique was applied in individual co-cultures of the pathogens Staphylococcus aureus and Staphylococcus epidermidis with Streptomyces roseosporus NRRL 15998. Using matrix-assisted laser desorption ionization (MALDI) imaging mass spectrometry, it was possible to observe three molecules $(\mathrm{m} / \mathrm{z} \quad 863,877$ and 891) that were responsible for the inhibition of both Staphylococcus pathogens. These stress-induced ions were subsequently identified as arylomycins, a class of broad-spectrum antibiotics that target type I signal peptidases [43].

\section{Metabolomics reveals naturally occurring microbial interactions}

Throughout thousands of years of evolution and survival adaptation, microorganisms have established all types of close relationships, including symbiosis, parasitism, competition and antagonism. Revealing the underlying molecular mechanisms involved in these 'invisible' interactions would presumably contribute to the discovery of new chemical entities with antibiotic activity, because virulence factors in their natural contexts might turn out to have beneficial applications in human anti-infective therapy [44]. Particularly, deciphering the microbial pathogenesis of some ecologically relevant systems, such as associations with plants, mushrooms, insects and sponges [45], opens new perspectives for the exploration for new antimicrobials, as exemplified by the discovery of the novel antibiotics, rhizoxin [46] and desoxyhavannahine [47] (Fig. 2). A very promising but yet quite unexplored reservoir for new antibiotics discovery is the insect-associated microbiome. As reviewed by Helge Bode [48], insects could be true pioneers as providers of antiinfectives for human beings. Paenilamicins (Fig. 2), a class of novel hybrid nonribosomal peptide/polyketide antibiotics, were discovered in the bee pathogen, Paenibacillus larvae. Bee larval co-infection assays revealed that the paenilamicins are employed by $P$. larvae to fight ecological niche competitors and are not directly involved in mortality of the bee larvae [49].

For cultivable symbionts, secondary metabolites produced in a natural context are probably different from those in a standard laboratory setting, because the growth condition considerably influences microbial secondary metabolism. Many cryptic biosynthetic gene clusters encoding molecules, remain silent in the laboratory setting due to the lack of appropriate environmental stimuli, and it is extremely challenging to activate their expression in the laboratory. More so, many symbiotic microbes are not even amenable to artificial cultivation. In other words, antibiotics that would occur due to in situ microbial interactions are impossible to be discovered in a traditional culture-dependent approach. Therefore, we need to mimic the natural setting to activate 'cryptic' antibiotics. The advances in metabolomics provide an opportunity to make what appeared to be impossible possible, using for example the above described IMS metabolome profiling techniques. The motile Gram-negative bacterium Janthinobacterium agaricidamnosum causes soft rot disease in the cultured button mushroom Agaricus bisporus. Driven by the hypothesis that the pathobiology of this mushroom pathogen was potentially correlated to antifungal search, MALDI-IMS was used for in situ profiling of the secondary metabolites harbored in the mushroom-associated ecological system of $A$. bisporus vs. J. agaricidamnosum. The visualization of a unique ion of $m / z 1181[\mathrm{M}+\mathrm{H}]^{+}$in the diseased region of the mushroom prompted the efforts to determine the structure of this compound and it was identified as an unprecedented cyclic lipopeptide product, jagaricin (Fig. 2). Jagaricin turned out to be a fungicide that is highly active against human pathogenic fungi including C. albicans, Aspergillus fumigatus and Aspergillus terreus at submicromolar concentrations. Notably, jagaricin production by the bacterium J. agaricidamnosum tended to occur exclusively in the 


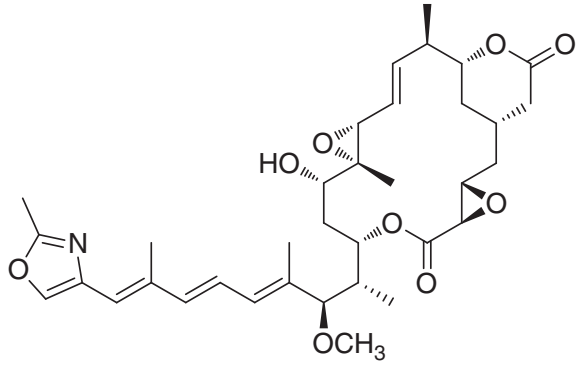

rhizoxin

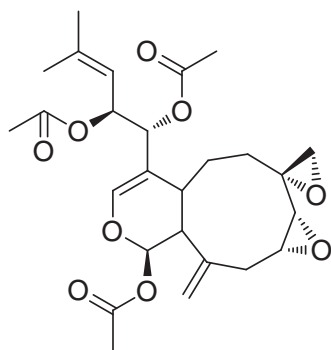

desoxyhavannahine

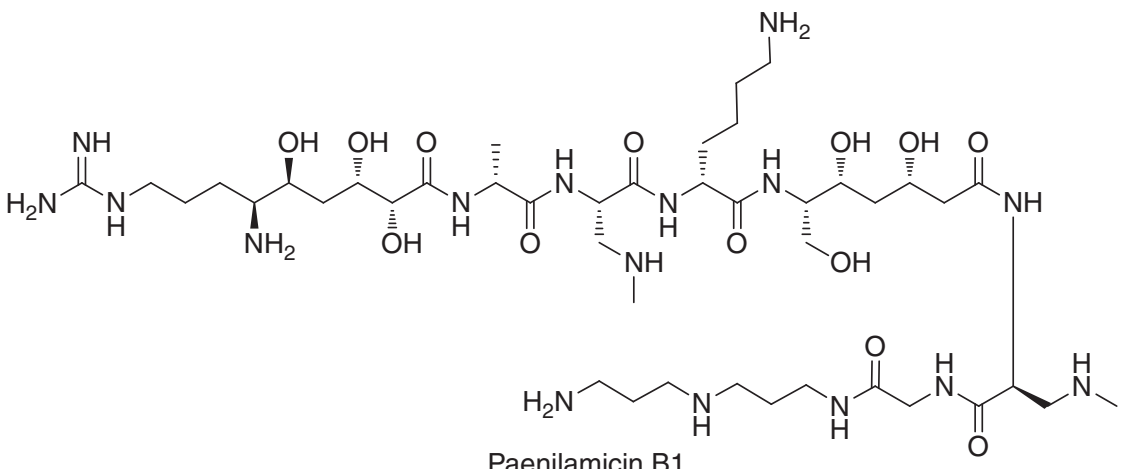

Paenilamicin B1

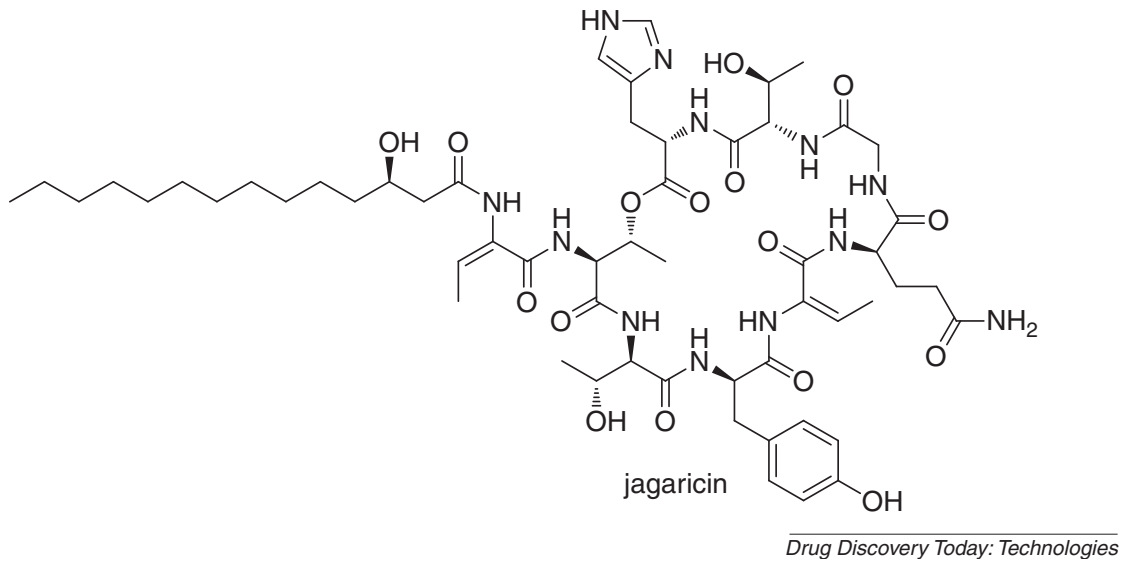

Figure 2. Structures of several novel antibiotics discovered through the investigation of microbial interactions.

mushroom (A. bisporus) and was otherwise impaired under a variety of laboratory cultivation conditions [50]. This example serves as a strong support for our arguments that understanding naturally occuring microbial pathogenesis is beneficial for antibiotic search; and that IMS-based metabolomics can be applied to reveal the cryptic antibiotics that are only produced in a particular natural environment.

Revealing cryptic new antibiotics in environmental samples might benefit from single cell metabolomics, a newly developed tool for metabolic analysis at cellular and subcellular level (recently reviewed in [51-53]). Our focus is onits potential use in unveiling the metabolic communication between symbionts and hosts. Based on the morphological phenotypes or other properties such as fluorescence, the selective metabolic analysis of certain microbial cells in environmental samples could be used to view the unique secondary metabolites generated by microorganisms during the naturally occuring interspecies interactions. For instance, halogen in situ hybridization-secondary ion mass spectroscopy (HISH-SIMS) has allowed the simultaneous identification and quantitation of metabolic activities of environmental microbial assemblage at single-cell level without the need for traditional microbial separation and laboratory cultivation [54]. Single-cell mass spectrometry (SCMS) is a rapidly 
growing field in analytical chemistry, and there are several examples of its applicability to different cells [51]. In this case, the possibility of accurate mass measurement and acquiring structural information as provided by tandem MS, facilitate the identification of numerous metabolites from a single cell.

\section{Conclusion}

Metabolomics methods provide an effective strategy for the discovery of new antibiotics from microbial sources. Allowing the comprehensive chemometric comparison of secondary metabolomes, it makes finding the proverbial needle in a haystack more realistic. The ability to measure the entire microbial metabolomes during symbiotic interactions can be crucial for the exploitation of these untapped reservoirs of anti-infectives [55]. Correlating gene expression profiles to fluctuations in the metabolome allows rapid identification of the gene cluster for the (known or unknown) bioactive molecule [56]. Thus, metabolomics complements (meta-)genomics methodology in accessing previously inaccessible natural products [57], and has the added advantage of delving into the biosynthetic potential of unculturable microorganisms when applied to in situ analysis of live, undamaged biological samples.

\section{References}

1. Rice LB. Federal funding for the study of antimicrobial resistance in nosocomial pathogens: no ESKAPE. J Infect Dis 2008;197:1079-81.

2. Cooper MA, Shlaes D. Fix the antibiotics pipeline. Nature 2011;472:32.

3. Payne DJ, Gwynn MN, Holmes DJ, Pompliano DL. Drugs for bad bugs: confronting the challenges of antibacterial discovery. Nat Rev Drug Discov 2007;6:29-40.

4. Bentley SD, et al. Complete genome sequence of the model actinomycete Streptomyces. Nature 2002;3:141-7.

5. Cruz-Morales P, et al. The genome sequence of Streptomyces lividans 66 reveals a novel tRNA-dependent peptide biosynthetic system within a metal-related genomic island. Genome Biol Evol 2013;5:1165-75.

6. Van den Berg MA, et al. Genome sequencing and analysis of the filamentous fungus Penicillium chrysogenum. Nat Biotechnol 2008;26:1161-8.

7. Yoon V, Nodwell JR. Activating secondary metabolism with stress and chemicals. J Ind Microbiol Biotechnol 2014;41:415-24.

8. Zhu H, et al. Triggers and cues that activate antibiotic production by actinomycetes. J Ind Microbiol Biotechnol 2014;41:371-86.

9. Kim HK, et al. NMR-based plant metabolomics: where do we stand, where do we go? Trends Biotechnol 2011;29:267-75.

10. Verpoorte R, et al. NMR-based metabolomics at work in phytochemistry. Phytochem Rev 2007;6:3-14.

11. Watrous J, et al. Mass spectral molecular networking of living microbial colonies. Proc Natl Acad Sci U S A 2012;109:1743-52. Reason:

Metabolomics combined with molecular networking were used to directly visualize the secondary metabolites produced by microorganisms on Petri dishes without complex sample pre-treatment.

12. Sidebottom AM, et al. Integrated metabolomics approach facilitates discovery of an unpredicted natural product suite from Streptomyces coelicolor M145. ACS Chem Biol 2013;8:2009-16.

13. Grkovic T, et al. NMR fingerprints of the drug-like natural-product space identify iotrochotazine A: a chemical probe to study Parkinson's disease. Angew Chem Int Ed Engl 2014;53:6070-4.

14. Williams DE, et al. Padanamides A and B, highly modified linear tetrapeptides produced in culture by a Streptomyces sp. isolated from a marine sediment. Org Lett 2011;13:3936-9.

15. Geng C-A, et al. LC-MS guided isolation of $( \pm)$-sweriledugenin A, a pair of enantiomeric lactones, from Swertia leducii. Org Lett 2014;16:370-3.
16. Kersten RD, et al. A mass spectrometry-guided genome mining approach for natural product peptidogenomics. Nat Chem Biol 2011;7:794-802

17. Pidot S, et al. Discovery of clostrubin, an exceptional polyphenolic polyketide antibiotic from a strictly anaerobic bacterium. Angew Chem Int Ed Engl 2014;53:7856-9.

18. Krug D, et al. Efficient mining of myxobacterial metabolite profiles enabled by liquid chromatography-electrospray ionisation-time-of-flight mass spectrometry and compound-based principal component analysis. Anal Chim Acta 2008;624:97-106.

19. Hou Y, et al. Microbial strain prioritization using metabolomics tools for the discovery of natural products. Anal Chem 2012;84:4277-83.

20. Carr G, et al. Microtermolides A and B from termite-associated Streptomyces sp. and structural revision of vinylamycin. Org Lett 2012;14:2822-5.

21. Hou Y, et al. Structure and biosynthesis of the antibiotic bottromycin D. Org Lett 2012;14:5050-3.

22. Wyche TP, et al. Forazoline A: marine-derived polyketide with antifungal in vivo efficacy. Angew Chem Int Ed 2014;53:11583-86. Reason: MS-based metabolomics was applied in strain prioritization from a microbial collection. MS-guided separation of target signals indicated by metabolomics analysis enabled new antibiotics discovery with novel skeleton and pronounced antimicrobial activity.

23. Seger C, et al. LC-DAD-MS/SPE-NMR hyphenation. A tool for the analysis of pharmaceutically used plant extracts: identification of isobaric iridoid glycoside regioisomers from Harpagophytum procumbens. Anal Chem 2005;77:878-85.

24. Glauser G, et al. Optimized liquid chromatography-mass spectrometry approach for the isolation of minor stress biomarkers in plant extracts and their identification by capillary nuclear magnetic resonance. J Chromatogr A 2008;1180:90-8

25. Bertrand S, et al. De novo production of metabolites by fungal co-culture of Trichophyton rubrum and Bionectria ochroleuca. J Nat Prod 2013;76:1157-65.

26. Wolfender J-L, et al. Phytochemistry in the microgram domain - a LCNMR perspective. Magn Reson Chem 2005;43:697-709.

27. Schroeder FC, Gronquist M. Extending the scope of NMR spectroscopy with microcoil probes. Angew Chem Int Ed Engl 2006;45:7122-31.

28. Bertrand S, et al. Metabolite induction via microorganism co-culture: a potential way to enhance chemical diversity for drug discovery. Biotechnol Adv 2014;32:1180-204.

29. Bertrand $S$, et al. Detection of metabolite induction in fungal co-cultures on solid media by high-throughput differential ultra-high pressure liquid chromatography-time-of-flight mass spectrometry fingerprinting. J Chromatogr A 2013;1292:219-28.

30. Ola A, Thomy D. Inducing secondary metabolite production by the endophytic fungus Fusarium tricinctum through coculture with Bacillus subtilis. J Nat Prod 2013;76:2094-9.

31. Cueto $\mathrm{M}$, et al. Pestalone, a new antibiotic produced by a marine fungus in response to bacterial challenge. J Nat Prod 2001;64:1444-6.

32. Oh D-C, et al. Induced production of emericellamides A and B from the marine-derived fungus Emericella sp. in competing co-culture. J Nat Prod 2007; 70:515-20.

33. Glauser G, et al. Differential analysis of mycoalexins in confrontation zones of grapevine fungal pathogens by ultrahigh pressure liquid chromatography/time-of-flight mass spectrometry and capillary nuclear magnetic resonance. J Agric Food Chem 2009;57:1127-34.

34. Scherlach K, et al. Symbiotic cooperation in the biosynthesis of a phytotoxin. Angew Chem 2012;124:9753-6. Reason: Secondary metabolite was jointly biosynthesized by two partners in an endosymbiotic relationship. The bacterial endosymbiont produces the rhizoxin macrolide backbone, which is tailored by an oxygenase provided by the fungal host. The referred cooperative biosynthesis is probably widespread in nature.

35. Watrous JD, Dorrestein PC. Imaging mass spectrometry in microbiology. Nat Rev Microbiol 2011;9:683-94

36. Krug D, Müller R. Secondary metabolomics: the impact of mass spectrometry-based approaches on the discovery and characterization of microbial natural products. Nat Prod Rep 2014;31:768-83.

37. Shih C-J, et al. Bringing microbial interactions to light using imaging mass spectrometry. Nat Prod Rep 2014;31:739-55. 
38. Hsu C-C, et al. Real-time metabolomics on living microorganisms using ambient electrospray ionization flow-probe. Anal Chem 2013;85:7014-8.

39. Watrous J, et al. Capturing bacterial metabolic exchange using thin film desorption electrospray ionization-imaging mass spectrometry. Anal Chem 2010;82:1598-600.

40. Liu W-T, et al. Imaging mass spectrometry of intraspecies metabolic exchange revealed the cannibalistic factors of Bacillus subtilis. Proc Natl Acad Sci U S A 2010;107:16286-90.

41. Hoe BC, et al. Enzymatic resistance to the lipopeptide surfactin as identified through imaging mass spectrometry of bacterial competition. Proc Natl Acad Sci U S A 2012;109:13082-87.

42. Traxler MF, et al. Interspecies interactions stimulate diversification of the Streptomyces coelicolor secreted metabolome. MBio 2013;4:1-12.

43. Liu W, et al. Imaging mass spectrometry and genome mining via short sequence tagging identified the anti-infective agent arylomycin in Streptomyces roseosporus. J Am Chem Soc 2011;133:18010-13.

44. Schmidt EW. Trading molecules and tracking targets in symbiotic interactions. Nat Chem Biol 2008;4:466-73.

45. Pidot SJ, et al. Antibiotics from neglected bacterial sources. Int J Med Microbiol 2014;304:14-22.

46. Partida-Martinez LP, Hertweck C. Pathogenic fungus harbours endosymbiotic bacteria for toxin production. Nature 2005;437:884-8.

47. Kelman D, et al. Chemical warfare in the sea: the search for antibiotics from Red Sea corals and sponges. Pure Appl Chem 2009;81:1113-21.

48. Bode HB. Insects: true pioneers in anti-infective therapy and what we can learn from them. Angew Chem Int Ed Engl 2009;48:6394-6.
49. Müller S, et al. Paenilamicin: structure and biosynthesis of a hybrid nonribosomal peptide/polyketide antibiotic from the bee pathogen Paenibacillus larvae. Angew Chem Int Ed Engl 2014;53:1-6.

50. Graupner $\mathrm{K}$, et al. Imaging mass spectrometry and genome mining reveal highly antifungal virulence factor of mushroom soft rot pathogen. Angew Chem Int Ed Engl 2012;51:13173-77. Reason: This research serves as a good example that IMS-based metabolomics reveals the cryptic antibiotics that are only produced in a particular natural environment.

51. Rubakhin SS, et al. Progress toward single cell metabolomics. Curr Opin Biotechnol 2013;24:95-104.

52. Trouillon R, et al. Chemical analysis of single cells. Anal Chem 2013;85:522-42.

53. Zenobi R. Single-cell metabolomics: analytical and biological perspectives. Science 2013;342:1201-11. Reason: Perspectives related to single-cell metabolomics are well reviewed in this paper.

54. Musat N, et al. A single-cell view on the ecophysiology of anaerobic phototrophic bacteria. Proc Natl Acad Sci U S A 2008;105:17861-66.

55. Perić-Concha N, Long PF. Mining the microbial metabolome: a new frontier for natural product lead discovery. Drug Discovery Today 2003;8:1078-84

56. Gubbens J, et al. Natural product proteomining, a quantitative proteomics platform, allows rapid discovery of biosynthetic gene clusters for different classes of natural products. Chem Biol 2014;21:707-18.

57. Brady SF, et al. Metagenomic approaches to natural products from freeliving and symbiotic organisms. Nat Prod Rep 2009;26:1488-503 